\title{
Application Effect of Nurse Patient Communication in Oncology Nursing
}

\author{
Xiaoyun Liu* \\ Weihai Central Hospital, Weihai 264400, Shandong Province, China \\ *Corresponding author: Xiaoyun Liu, xt666999@yeah.net
}

\begin{abstract}
Objective: To explore the clinical significance of strengthening nurse patient communication in oncology department. Methods: The study was carried out in our hospital. Taking 100 patients selected from May 2020 to May 2021 as an example, all patients were cancer patients. When they were treated, they cooperated with nursing, carried out grouping experiments, adopted different nursing methods, and compared and analyzed the recovery of patients under different nursing interventions. Results: There were significant differences in nursing satisfaction and compliance between the experimental group and the control group, expressed as $\mathrm{P}<0.05$. In contrast, the experimental group had higher satisfaction, better compliance and better effect. Conclusion: Strengthening nurse patient communication in oncology patients is helpful to improve patients' understanding, improve patients' bad mood and promote patients' compliance. It has positive significance and remarkable effect on patients.
\end{abstract}

Keywords: Nurse patient communication; Oncology department; Nursing effect

Publication date: November 2021; Online publication: November 30, 2021

\section{Introduction}

In the actual development, the incidence rate of cancer patients is gradually increasing. Patients with this disease will have various bad emotions, including depression and anxiety, which will lead to the increase difficulty of nursing work ${ }^{[1]}$. In this process, it is particularly important to strengthen nurse-patient communication, which can avoid the impact on the treatment effect and prognosis recovery of patients, ensure patient's compliance, and reduce the incidence of doctor-patient disputes. Therefore, nurses need to start from the actual situation, give patients active and effective nursing intervention, strengthen communication and improve patients' trust ${ }^{[2]}$. On this basis, taking patients in our hospital as an example, this study implemented the nursing mode of routine nursing and strengthening nurse-patient communication respectively, and compared and analyzed its clinical application effect.

\section{Data analysis and research methods}

\subsection{General information}

The comparative experiment was carried out in our hospital from May 2020 to May 2021. 100 oncology patients received during this period were taken as examples, including 54 males and 46 females. The age of the patients ranged from 52 to 78 years, with an average age of $(65.45 \pm 2.34)$ years. Among them, there were 33 cases of gastric cancer, 23 cases of breast cancer, 25 cases of lung cancer and 19 cases of colorectal cancer. The patients in this study were grouped based on the principle of average distribution. Under statistics, the age and gender of the two groups were similar, $\mathrm{P}>0.05$. The difference between the two groups was the treatment method, and they were named the experimental group and the control group.

(1) Inclusion criteria: All patients were cancer patients. The patients were informed of the study and 
agreed to participate in this research. The patients were given Montreal cognitive assessment scale to evaluate their cognitive status. The scores of patients were 10-26. The study was approved by the hospital ethics committee.

(2) Exclusion criteria: The patient has cognitive impairment. Patients has a history of mental abnormality; The patient has communication barriers. The patients' data are incomplete.

\subsection{Research methods}

The 50 patients in the control group received routine nursing. The nurses managed the patients, strengthened dietary intervention, regularly observed the patients' condition, and gave patients health education. On the basis of routine nursing intervention, 50 patients in the experimental group increased nurse-patient communication for patients. The specific implementation process is as follows:

Communication when admission: In general, patients have relatively large emotional fluctuations after learning that they have tumor. Patients are usually accompanied by depression, anxiety and other symptoms. Nurses need to strengthen communication with patients, give patients encouragement and support, strengthen understanding of patients, master patients' psychological changes, and help patients go through various procedures after admission as well as let patients feel warm. For newly admitted patients, nurses need to meet patients with a dignified, appropriate and gentle attitude, introduce the surrounding environment to patients and their families, ensure that patients adapt to hospital life as soon as possible, establish a good relationship with patients, and ensure patients' trust, so as to promote the smooth development of various work.

Communication during treatment: For cancer patients, the treatment methods are usually surgery, radiotherapy and chemotherapy. When giving patients treatment intervention, patients are prone to worry, fear and other emotions. Nurses need to master the psychological emotions of patients, explain the necessity of treatment, treatment effect and possible adverse reactions for patients, improve patients' awareness and avoid excessive worry, explain the successful treatment cases for patients, improve their treatment selfconfidence, understand the family situation of patients, as well as strive for some preferential schemes for patients, so as to reduce the psychological pressure of patients and promote patients to actively cooperate with treatment. At the same time, nurses need to strengthen room patrol, observe patients, pay attention to the changes of patients' condition, actively manage patients, listen to patients' complaints, and ensure that patients are treated in time when adverse symptoms are found, so as to avoid adverse reactions and ensure the safety of patients.

Communication with patients with advanced cancer: For patients with advanced cancer, most of them have limited survival time, so nurses need to pay more attention to them, carry out psychological intervention on the patient's family members, enlighten the family members, promote the family members to correctly understand death and disease, urge the family members to accompany the patients, reduce the patient's tension, give family support to the patients, and improve the patient's quality of life. In the process of nursing at home, nurses need to strengthen communication with patients, clarify the needs of patients, and meet the needs of patients as much as possible.

\subsection{Observation indexes}

The nursing compliance of the two groups was compared. The daily cooperation degree of the patients was mainly observed, including the compliance with radiotherapy and chemotherapy, taking drugs on time, good mentality and so on. The evaluation scale was divided into three items, including complete compliance, compliance and non-compliance. The number of patients with compliance was counted, complete compliance + compliance $=$ compliance.

Compare the nursing satisfaction of the two groups of patients, and apply the self-made satisfaction 
questionnaire in our hospital. The full score of the questionnaire is 100 points. A high score indicates that the patient's satisfaction is good. It is sorted into three indicators, which are very satisfied (more than 90 points), satisfied (70-90 points) and dissatisfied (less than 70 points). Excluding dissatisfaction, it is the total satisfaction.

\subsection{Statistical methods}

For data statistics, SPSS20.0 software is mainly used. For the calculation of measurement data, the result is mainly $(\bar{x} \pm s)$, and the verification is mainly $t$ value. For the statistics of counting data, the comparison result is counted by $(\mathrm{n}, \%)$, and the verification is expressed by $\mathrm{X}^{2}$ value. When the result shows that the data ratio is 0.05 hours, it indicates that the calculation of this study is meaningful.

\section{Results}

\subsection{Comparison of patient's compliance}

Comparing the compliance of the two groups, the compliance of the experimental group was $92.00 \%$, significantly higher than $80.00 \%$ of the control group, with significant difference $(\mathrm{P}<0.05)$. See Table 1 for details.

Table 1. Comparison of treatment compliance differences [n (\%)]

\begin{tabular}{lcccc}
\hline Group & Complete compliance & Compliance & Non-compliance & Compliance \\
\hline Experimental group $(\mathrm{n}=50)$ & $28(56.00)$ & $18(36.00)$ & $4(8.00)$ & $46(92.00)$ \\
Control group $(\mathrm{n}=50)$ & $15(30.00)$ & $25(50.00)$ & $10(20.00)$ & $40(80.00)$ \\
$\mathrm{X}^{2}$ & & & & 6.442 \\
$\mathrm{P}$ & & & & $<0.05$ \\
\hline
\end{tabular}

\subsection{Nursing satisfaction}

There was significant difference in nursing satisfaction between the two groups $(\mathrm{P}<0.05)$, and the satisfaction of patients in the experimental group was higher, as shown in Table 2.

Table 2. Comparison of nursing satisfaction between the two groups [n (\%)]

\begin{tabular}{lcccc}
\hline Group & Very satisfied & Satisfied & Dissatisfied & Satisfaction \\
\hline Experimental group $(\mathrm{n}=50)$ & $36(72.00)$ & $12(24.00)$ & $2(4.00)$ & $48(96.00)$ \\
Control group $(\mathrm{n}=50)$ & $24(48.00)$ & $19(38.00)$ & $7(14.00)$ & $43(86.00)$ \\
$\mathrm{X}^{2}$ & & & & 7.354 \\
$\mathrm{P}$ & & & & $<0.05$ \\
\hline
\end{tabular}

\section{Discussion}

In recent years, China's economic construction has been gradually promoted, and the development capacity of various technologies has been gradually improved. On this basis, the level of medical technology has been improved, and the tumor detection rate has been continuously improved. Tumor is an important disease that poses a serious threat to people's life and health. The treatment of patients is an important method to prolong the survival time of patients ${ }^{[3]}$. However, in the actual development, due to the relatively large damage of treatment methods to patients and the low understanding of their own diseases, patients are prone to adverse emotions such as anxiety and depression, reduce cooperation compliance, which is not conducive to the prognosis of patients. According to the results of relevant research data, strengthening 
nurse-patient communication among cancer patients can alleviate the relationship between patients and nurses, which is of positive significance to improve patient satisfaction ${ }^{[4]}$. At the same time, strengthening nurse patient communication can promote the communication between nurses and patients, eliminate the barriers between them, improve the patients' trust in nurses, and fully reflect the nurses' working value, which is conducive to the hospital to improve its image ${ }^{[5]}$.

In the process of promoting nurse-patient communication, this study strictly follows the concept of humanized nursing, starting from the actual situation of the patient, combining the specific situation of the patient, and adopting different communication methods for different patients. For newly admitted patients, it is necessary to introduce the hospital environment, the attending doctor, etc. For patients with insufficient understanding of diseases and treatment methods, health education should be given to patients during treatment to improve their understanding. For patients with advanced malignant tumor, health education should be given to their families to encourage them to accompany patients more and ensure that patients feel the warmth of their families ${ }^{[6]}$. Different communication for different nurses can promote the targeted improvement of nursing effect, which is of positive significance to ensure patients' recovery and improve patients' satisfaction ${ }^{[7]}$. After the completion of the experiment, the results show that the patients in the experimental group have higher nursing satisfaction and better patient compliance, which are significantly different from the control group, indicating the clinical significance of strengthening nurse-patient communication in tumor patients.

In conclusion, strengthening nurse-patient communication among oncology patients has significant effect, which can promote patients' awareness and ensure patients' compliance. It is of positive significance to improve patients' nursing satisfaction, has significant clinical application value and can be actively promoted.

\section{Disclosure statement}

The author declares no conflict of interest.

\section{References}

[1] Wang L, Xu H, 2019, Analysis of Application Effect of Nurse Patient Communication in Oncology Nursing. Journal of Clinic Nursing's Practicality, 4(19): 97-98.

[2] Gu Y, 2019, Analysis of Application Effect of Nurse Patient Communication in Oncology Nursing. Journal of Clinic Nursing's Practicality, 004(038): 126-126.

[3] Huang S, Shi Y, 2019, Discussion on Application of Nurse Patient Communication in Neurosurgical Nursing. Food Science.

[4] Zhang L, Huang J, 2019, Analysis of Application Effect of Nurse Patient Communication in Pediatric Surgery Nursing. Journal of Frontier of Medicine, 9(6): 219-220.

[5] Pan Y, 2019, Discussion on Application of Nurse Patient Communication in Cardiological Nursing. Psychological Doctor, 25(002): 350-351.

[6] Du H, 2019, Analysis of Application Effect of Humanized Nurse Patient Communication in Internal Medical Nursing. Journal of Frontier of Medicine, 2019, 009(007): 189.

[7] Ma Y, 2019, Application Effect of Good Nurse Patient Communication in Surgical Outpatient Nursing. Psychologies, 14 (04): 96-96. 